\title{
EFFECTS OF SECOND-APPROXIMATION OF BOUNDARY LAYER THEORY IN THREE-DIMENSIONAL FLOW OVER BODIES WITH A LARGE ASPECT RATIO AT SMALL ANGLES OF INCIDENCE
}

\author{
E. A. Lipchinskii, G. A. Tirskii, and S. V. Utyuzhnikov
}

UDC 532.526.2:533.6.011.55

\begin{abstract}
The influence of the second-approximation effects of laminar boundary layer theory on the heat transfer in three-dimensional hypersonic flow over blunt cones with a large aspect ratio is investigated numerically.
\end{abstract}

The influence of the second-approximation effects of boundary layer theory in the neighborhood of the stagnation point was first investigated in detail in [1]. In that study the principal second-approximation effects caused by the terms of order $O\left(\mathrm{Re}^{-1 / 2}\right)$, which are not involved in the Prandtl equations, were identified. It was noted that the effects of entropy layer absorption by the boundary layer predominate over all the other second-order effects. In [2] it was shown that the contribution of the entropy layer absorption effect to the heat flux on the surface of an orbiter can reach $50 \%$. The entropy layer absorption manifests itself at large distances from the bluntness. Various approximate approaches were developed for taking the entropy layer absorption into account within the framework of the classical Prandtl model for bodies with a large aspect ratio. These approaches involve both analytical studies of the problem and various engineering methods based on taking the vorticity on the outer edge of the boundary layer approximately into account (see, for example, [3-7]).

The system of equations of the complete viscous shock layer contains all the terms of the complete Navier-Stokes equations which contribute to the second approximation of boundary layer theory and makes it possible to determine with quantitative accuracy their total contribution. In study [8] the influence of the effects in question was determined for hypersonic axisymmetric flow over blunt cones with a large aspect ratio. It was found that the entropy layer absorption effect can lead to substantial changes in the heat transfer and friction coefficients (by up to 100\%) in the zone of intense absorption, as compared with the results for the first-approximation boundary layer (classical theory). It was noted that cooling the surface of the body leads to a decrease in the influence of the entropy layer absorption effect. The applicability of approximate methods of taking the entropy layer absorption into account was studied in [3-7]. In [9] the contribution of the second-approximation effects of boundary layer theory was investigated in the case of axisymmetric air flow over blunt bodies assuming equilibrium dissociation and ionization reactions in the shock layer.

In the present paper the combined influence of the second-approximation effects in three-dimensional unseparated flow over long blunt cones is investigated. In contrast to [8], here we shall investigate a three-dimensional flow. The solution over the entire domain between the surface of the body and the surface of the shock wave, including subsonic flow regions, is found in a unified manner using a numerical method based on global iterations [10].

\section{FORMULATION OF THE PROBLEM AND METHOD OF SOLUTION}

We will consider an unseparated flow over long blunt cones at small angles of incidence. In order to describe the gas flow between the surface of the body and the surface of the shock wave we use the system of three-dimensional viscous boundary layer equations, which contains all the terms of the complete system of Navier-Stokes equations up to and including the terms of order $O\left(\operatorname{Re}^{-1 / 2}\right)$. We write this system of equations in an orthogonal coordinate system moving with the surface of the body in the usual way ( $x$ is the length of body contour generator, $y$ is the distance to the surface of the body along the normal, and $\varphi$ is the meridional angle reckoned from the spreading plane) [11]:

$$
\frac{\partial}{\partial x}\left(H_{2} \rho u\right)+\frac{\partial}{\partial \varphi}\left(H_{1} \rho w\right)+\frac{\partial}{\partial y}\left(H_{1} H_{2} \rho v\right)=0
$$

Moscow. Translated from Izvestiya Rossiiskoi Akademii Nauk, Mekhanika Zhidkosti i Gaza, No. 2, pp. 57-64, March-April, 1995. Original article submitted October 29, 1993. 


$$
\begin{gathered}
\rho\left[D u-\frac{w^{2}}{H_{1} H_{2}} \frac{\partial H_{2}}{\partial x}+\frac{u w}{H_{1} H_{2}} \frac{\partial H_{1}}{\partial \varphi}+\frac{u v}{H_{1}} \frac{\partial H_{1}}{\partial y}\right]=-\frac{1}{H_{1}} \frac{\partial P}{\partial x}+\frac{1}{H_{1}^{2} H_{2} \operatorname{Re} e_{-}} \frac{\partial}{\partial y}\left[H_{1}^{3} H_{2} \mu \frac{\partial}{\partial y}\left(\frac{u}{H_{1}}\right)\right] \\
\rho\left[D w+\frac{w u}{H_{1} H_{2}} \frac{\partial H_{2}}{\partial x}-\frac{u^{2}}{H_{1} H_{2}} \frac{\partial H_{1}}{\partial \varphi}+\frac{w v}{H_{1}} \frac{\partial H_{2}}{\partial y}\right]=-\frac{1}{H_{2}} \frac{\partial P}{\partial \varphi}+\frac{1}{H_{1} H_{2} \operatorname{Re}_{\infty}} \frac{\partial}{\partial y}\left[H_{1} H_{2}^{3} \mu \frac{\partial}{\partial y}\left(\frac{w}{H_{2}}\right)\right] \\
\rho D H=\frac{1}{H_{1} H_{2}} \frac{\partial}{\partial y}\left\{\frac{H_{1} H_{2} \mu}{\sigma \operatorname{Re}_{\infty}}\left[\frac{\partial H}{\partial y}+\frac{V_{\infty}^{2}(\sigma-1)}{2 H_{\infty}} \frac{\partial\left(u^{2}+w^{2}\right)}{\partial y}-\frac{\sigma u^{2} V_{-}^{2}}{H_{\infty} H_{1}} \frac{\partial H_{1}}{\partial y}-\frac{\sigma H_{1}}{\partial y} u^{2}-\frac{1}{H_{2}} \frac{\partial H_{-}}{\partial y} w_{-}^{2} \frac{\partial H_{2}}{\partial y}\right]\right) \\
D=\frac{u}{H_{1}} \frac{\partial}{\partial x}+\frac{\partial P}{H_{2}} \frac{\partial}{\partial \varphi}+v \frac{\partial}{\partial y}, \quad H=h+\frac{v_{\infty}^{2}}{2 H_{-}}\left(u^{2}+w^{2}+v^{2}\right), \quad h=\frac{\gamma P V_{-}^{2}}{(\gamma-1) \rho H_{-}}
\end{gathered}
$$

Here, $u, v$, and $w$ are the physical components of the velocity vector in the $x, \varphi$, and $y$ directions; $R(x, \varphi)$ is the radius of curvature of the body surface; $\operatorname{Re}_{-}$is the Reynolds number; $x(x, \varphi)=1 / R(x, \varphi)$ is the curvature of the surface of the body; $\sigma$ is the Prandtl number; $H_{1}$ and $H_{2}$ are the Lamé coefficients; $\alpha$ is the angle of inclination of the generator of the body to the axis of symmetry; $r_{w}$ is the distance from a point on the surface of the body to its axis.

In the system of equations (1.1) the quantities are nondimensionalized as follows: the velocity vector components have been divided by the free-stream velocity $V_{-}$the pressure $P$ by $\rho_{-} V_{-}^{2}$, the total enthalpy $H$ by $H_{-}$and the quantities having the dimension of length by the bluntness radius $R(0)$.

In the case of a perfect gas the viscosity coefficient $\mu$ is assumed to be a known function of the absolute temperature $T$. In the calculations we used Sutherland's formula.

The system of equations (11) is closed using the following boundary conditions. On the shock wave, considered as a surface of a strong discontinuity, the generalized Rankine-Hugoniot conditions are imposed [12]. In the coordinate system $(x, \varphi, y)$ these conditions have the form:

$$
\begin{aligned}
& u_{s}=u_{s} \tan \beta_{s}+w_{s} \tan \gamma_{3}+K_{s} \cdot V_{s}(3) \\
& P_{s}=\frac{1}{\gamma M_{\infty}^{2}}+\frac{V_{-\infty}^{2}(3)\left(1-K_{s}\right)}{1+\tan ^{2} \beta_{s}+\tan ^{2} \gamma_{s}} \\
& u_{s}=V_{-}(1) \cos ^{2} \beta_{s}-\frac{w_{s}}{2} \sin 2 \beta_{s} \tan \gamma_{s}-\frac{K_{s}}{2} V_{-}(3) \sin 2 \beta_{s}+ \\
& \frac{\mu_{s}}{\operatorname{Re}_{\infty} V_{s}(3)}\left(\frac{\partial u}{\partial y}-\frac{u}{H_{1}} \frac{\partial H_{1}}{\partial y}\right)_{s} \\
& w_{s}=V_{-}(2) \cos ^{2} \gamma_{s}-\frac{u_{s}}{2} \sin 2 \gamma_{s} \tan \beta_{s}-\frac{K_{s}}{2} V_{-}(3) \sin 2 \gamma_{s}+ \\
& \frac{\mu_{s}}{\operatorname{Re}_{-} V_{-}(3)}\left(\frac{\partial w}{\partial y}-\frac{w}{H_{2}} \frac{\partial H_{2}}{\partial y}\right)_{s} \\
& H_{s}=1+\frac{\mu_{s}}{\sigma \operatorname{Re}_{\infty} V_{\infty}(3)}\left(\frac{\partial H}{\partial y}+\frac{V_{\infty}^{2}(\sigma-1)}{2 H_{-}} \frac{\partial\left(u^{2}+w^{2}\right)}{\partial y}-\frac{\sigma u^{2} V_{\omega}^{2}}{H_{\alpha} H_{1}} \frac{\partial H_{1}}{\partial y}-\frac{\sigma w^{2} V_{-}^{2}}{H_{\infty} H_{2}} \frac{\partial H_{2}}{\partial y}\right) \\
& \tan \beta_{s}=\frac{1}{H_{1 s}} \frac{\partial y_{s}}{\partial x}, \quad \tan \gamma_{s}=\frac{1}{H_{2 s}} \frac{\partial y_{s}}{\partial \varphi}, \quad K_{s}=\frac{1}{\rho_{s}}
\end{aligned}
$$

Here, the subscript $s$ denotes the quantities behind the surface of the shock wave; $V_{-}(i), i=1,2,3$, are the free- 


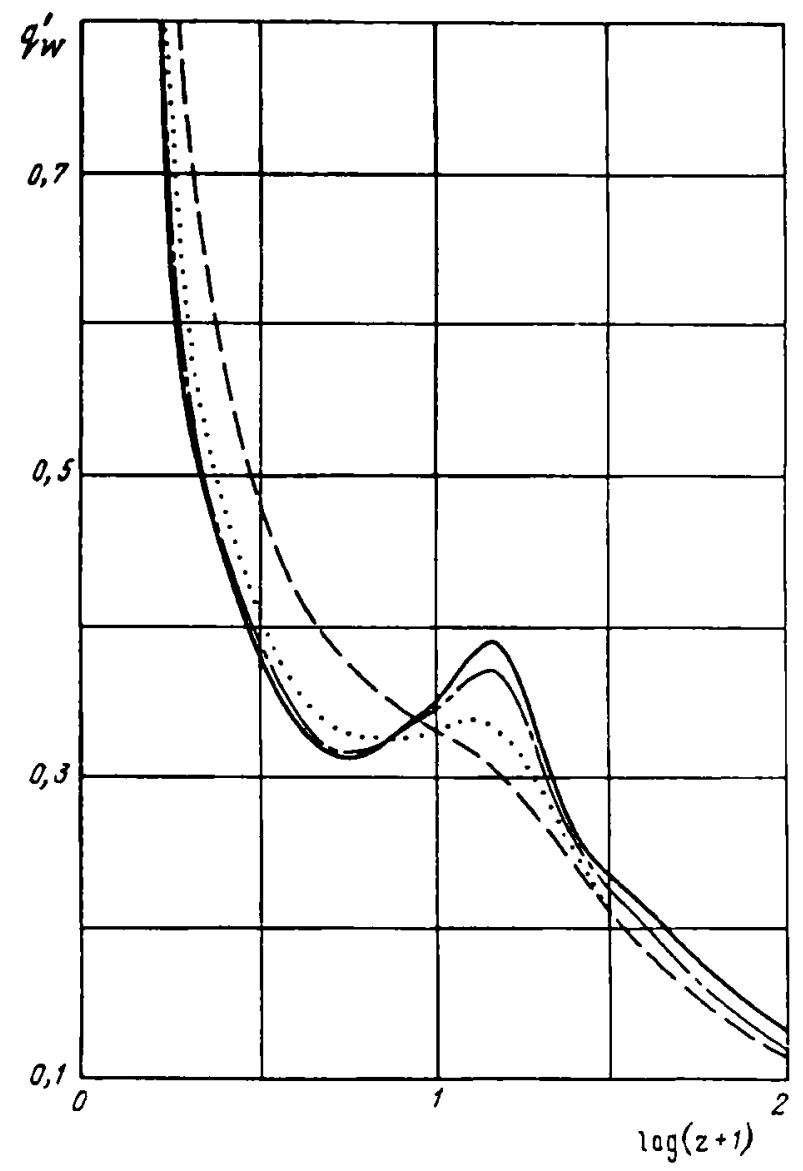

Fig. 1

stream velocity vector components in the coordinate system $(x, \varphi, y) ; \beta_{s}$ and $\gamma_{s}$ are the angles of inclination between the surface of the body and the surface of the shock wave; $y_{s}=y_{s}(x, \varphi)$ is the shock wave standoff distance. As boundary conditions, on the surface of the body the no-slip, no-flow, and cooled wall conditions are imposed:

$$
u(x, \varphi, 0)=w(x, \varphi, 0)=v(x, \varphi, 0)=0, \quad H(x, \varphi, 0)=H_{w}^{0}
$$

Here, the subscript $w$ denotes the quantities on the body surface.

The problem is solved in the domain between the surfaces of the body and the detached shock wave: $0 \leq x \leq x_{k}$, $0 \leq \varphi<2 \pi, 0 \leq y \leq y_{s}$. On the surface $x=x_{k}$ "soft" boundary conditions are imposed.

For solving the system (1.1)-(1.3) we used the method of asymptotic expansion in terms of a small parameter, namely, the angle of incidence. The system of equations for the expansion coefficients is solved by the global iteration method [10]. This numerical method, which was described in detail in [11], makes it possible to reduce the computer time by a factor of approximately 100 as compared with the stabilization methods used in the complete three-dimensional formulation. The accuracy of the method was confirmed in [11] by comparison with experimental and calculated data. In order to integrate the system of governing equations numerically we used a difference scheme of second-order approximation with respect to the longitudinal and fourth-order approximation with respect to the transverse coordinate. In the direction normal to the body we used a variable step distribution of the difference grid, the steps being chosen at each point in accordance with the variation of the function in its neighborhood. The computational grid consisted of 50 nodes in the direction transverse to the body.

\section{RESULTS}

In order to investigate the second-approximation effects of boundary layer theory we calculated numerically the flow over spherically blunt cones with a length equal to 150 bluntness radii over a broad range of free-stream Reynolds 


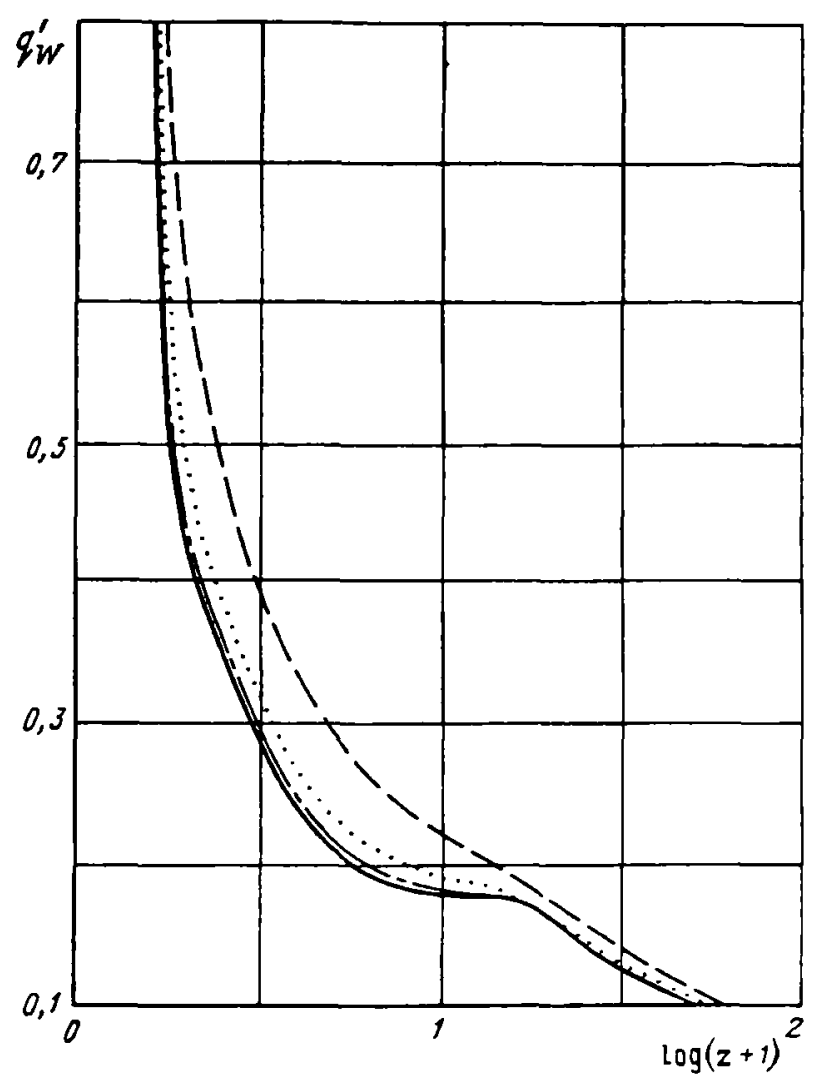

Fig. 2

numbers $\mathrm{Re}_{-}$for various Mach numbers $\mathrm{M}_{-}$, temperature factors $t_{w}$, and cone half-angles $\alpha_{0}$. The influence of the second-order effects was determined using the heat flux distribution

$$
q_{w}^{\prime}=q_{w} \sqrt{\operatorname{Re}_{\infty}} \quad q_{w}=\lambda \frac{\partial T}{\partial y}\left[\rho_{-} V_{\infty} c_{p} T_{0}\right]^{-1}
$$

Here, $\lambda$ is the thermal conductivity, and $T_{0}$ is the stagnation temperature of the flow. $A s \operatorname{Re}_{\infty} \rightarrow \infty q_{w}{ }^{\prime}$ reaches a limiting value which does not depend on $\mathrm{Re}_{\text {. }}$ and corresponds to the solution of the equations of the classical boundary layer. In the case of a fairly large finite value of $\mathrm{Re}_{-}$the corresponding difference between the distribution $q_{w}{ }^{\prime}(x)$ and the limiting one makes it possible to determine the total contribution of the second-approximation effects of boundary layer theory. The results of calculations made for the values $\operatorname{Re}_{\infty}=10^{3}, 10^{4}, 10^{5}$, and $10^{6}$ are presented. When $\operatorname{Re}_{\infty}=10^{3}$ the shock layer is completely viscous and there is no boundary layer as such. At the same time, the curves for $\operatorname{Re}_{-}=10^{5}$ and $10^{6}$ differ only slightly, which corresponds to the establishment of the boundary layer asymptotics; when $\operatorname{Re}_{-}=10^{7}$ the graph of $q_{w}{ }^{\prime}$ is not visually distinguishable from the graph for $\operatorname{Re}_{-}=10^{6}$.

In Fig. 1 we have plotted the heat flux distribution on the windward surface of a $15^{\circ}$-cone for $\mathbf{M}_{-}=20$, $t_{w}=0.5$, and angle of incidence $\theta=5^{\circ}$ in the spreading plane (here, $z$ is the coordinate along the axis of the cone). Here and in what follows, the broken, dotted, chain, and continuous curves correspond to $\operatorname{Re}_{-}=10^{3}, 10^{4}, 10^{5}$, and $10^{6}$, respectively. As can be seen from the figure, the main "stratification" of the curves corresponds to the values of $z=1-6$. In the neighborhood of 10 radii there is a small zone in which $q_{w}{ }^{\prime}$ practically does not depend on $\mathbf{R e}_{-}$. Then there is an inversion in the distribution of $q_{w}{ }^{\prime}$ as a function of $\operatorname{Re}$. with higher values of the heat flux $q_{w}{ }^{\prime}$ corresponding to higher values of $\operatorname{Re}$. At distances of more than 100 bluntness radii for $\mathrm{Re}_{-} \geq 1000$ the high-entropy layer is almost completely absorbed by the boundary layer and the corresponding values of $q_{w}{ }^{\prime}$ differ only slightly. When there is no angle of incidence, inversion of the heat flux is not observed (Fig. 2). The inversion effect appears to be attributable to the influence of secondary flows caused by the three-dimensional nature of the flow.

The distribution of $q_{w}{ }^{\prime}$ over the Reynolds numbers also remains monotonic on the leeward side of the cone, although in this zone the applicability of the small parameter method is conjectural. In order to eliminate the influence of errors associated with the approximation of the method used, we considered the analogous family of curves for the 


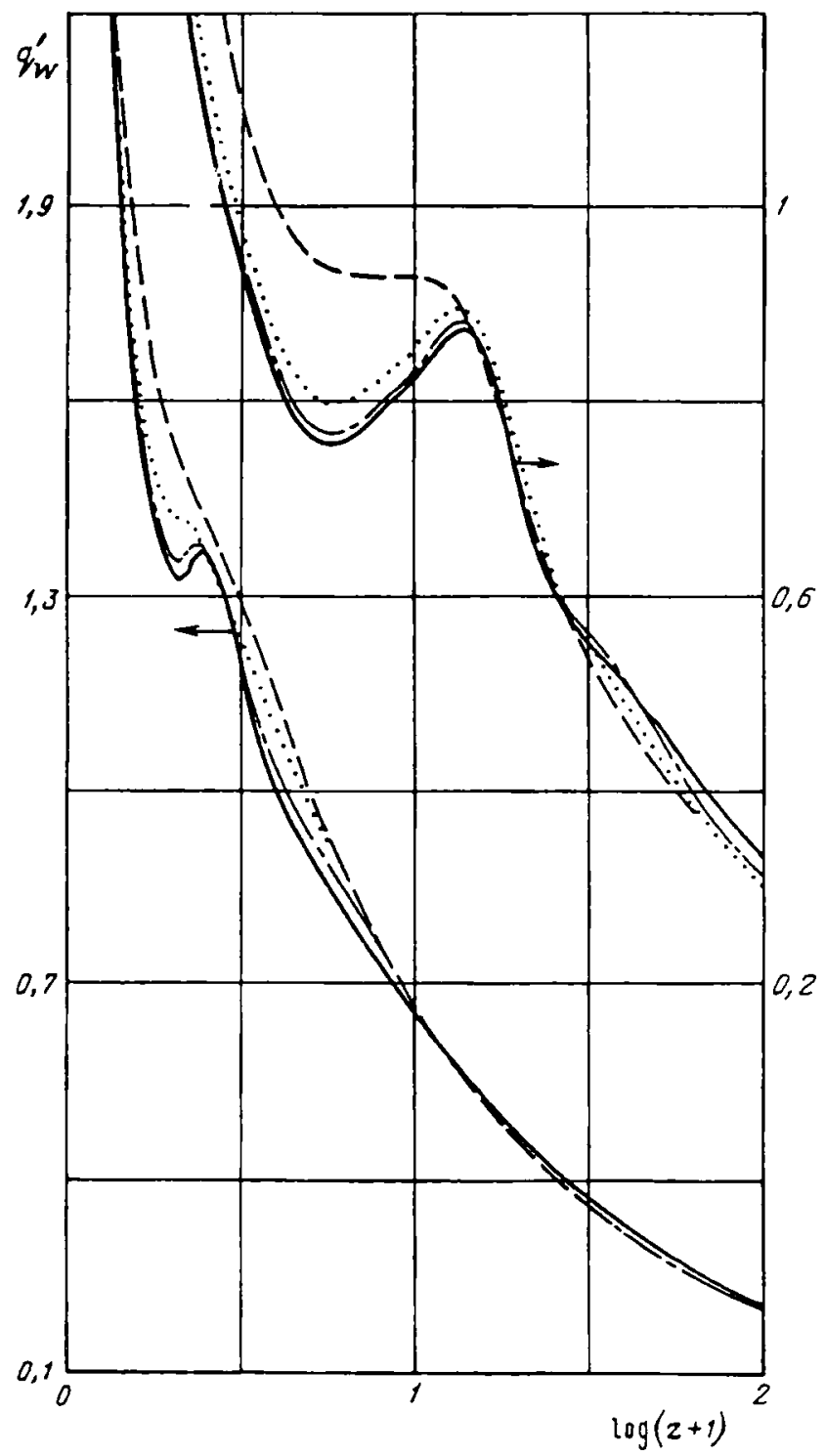

Fig. 3

angle of incidence $\theta=2^{\circ}$. In this case the conclusions were qualitatively the same, but the point of intersection of the curves (on the windward side) was displaced upstream by approximately 10 radii. In the case of a cold wall $\left(t_{w}=0.05\right.$ ) the entropy layer absorption is more weakly expressed, the inversion of the heat flux being observed later (Fig. 3). In Fig. $3 a$ the cone and free-stream parameters are the same as in Fig. 1, except for $t_{w}$.

In Fig. $3 b$ we have shown the distribution of $q_{w}{ }^{\prime}$ for windward-side flow over a $35^{\circ}$-cone with $M_{-}=20, t_{w}=0.5$, and $\theta=10^{\circ}$. The increase in the cone angle leads to the entropy layer absorption coming earlier and, starting from 10 radii, the solution corresponds to the classical boundary layer asymptotics. When $\mathrm{Re}_{-}>10^{4}$ in the neighborhood of 1.5 $R_{0}$ there is a clearly defined second extremum of the heat flux, but the inversion of $q_{w}{ }^{\prime}\left(\mathrm{Re}_{-}\right)$is more weakly expressed. It is of interest to note that, starting from $\operatorname{Re}_{-}=10^{4}$, the quantity $q_{w}{ }^{\prime}$ practically does not change in the neighborhood of the local extremum. On the leeward side the solution differs qualitatively in that the intense entropy layer absorption comes considerably later - in the vicinity of 50 radii. In the case of flow over the same cone but at an angle of incidence of $5^{\circ}$ the entropy layer absorption on the windward side is displaced into the neighborhood of 20 bluntness radii.

The flow over a cone with the same parameters as in Fig. 1 but for $M_{-}=5$ (Fig. 4) is characterized by the fact that, along with a decrease in the heat flux, the influence of the entropy layer absorption is weakened. In this case, starting from $\mathrm{Re}_{-}=10^{5}$, the solution corresponds to the classical boundary layer asymptotics. A second extremum of $q_{w}{ }^{\prime}$ is not 


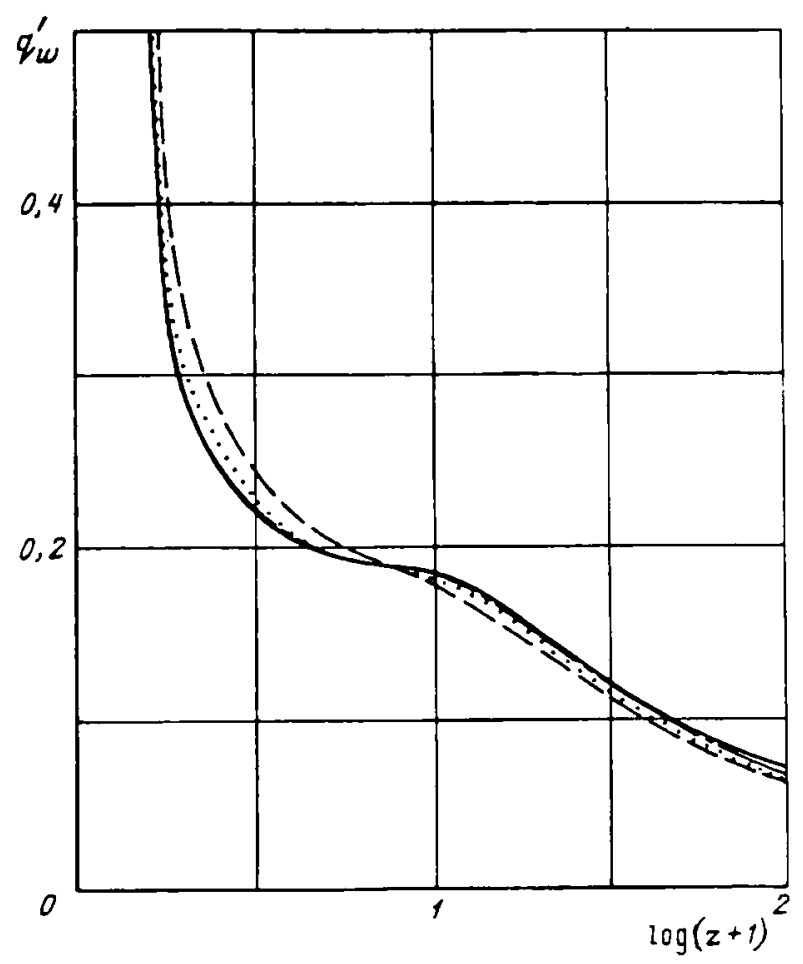

Fig. 4

formed, although there is a weak inversion of $q_{w}{ }^{\prime}\left(\operatorname{Re}_{-}\right)$at $z>10$.

\section{REFERENCES}

1. M. D. Van Dyke, "Second-order compressible boundary layer theory with application to blunt bodies in hypersonic flow," in: Hypersonic Flow Research, Academic Press, New York (1962).

2. W. D. Goodrich, C. P. Li, C. K. Houston, et al., "Numerical computations of orbiter flowfields and laminar heating rates," J. Spacecr. Rockets, 14, 257 (1977).

3. Yu. G. El'kin. Yu. N. Ermak, I. I. Lipatov, and V. Ya. Neiland, "Entropy layer absorption on a blunt cone in a hypersonic viscous gas flow," Uch. Zap. TsAGI, 14, 18 (1983).

4. N. P. Kolchina, A. I. Pyatnova, and E. E. Solodkin, "Effect of entropy layer absorption on the characteristics of long blunt bodies for various forms of boundary layer flow," Tr. TsAGJ, No. 2107, 161 (1981).

5. P. E. Babikov and V. A. Bashkin, "Calculation of the laminar boundary layer on slender bodies with allowance for entropy layer absorption," Tr. TSAGI, No. 1909, 33 (1978).

6. V. V. Lunev, "Mass-average value method for the boundary layer in an external flow with transverse nonuniformity," Izv. Akad. Nauk SSSR, Mekh. Zhidk. Gaza, No. 1, 127 (1967).

7. B. A. Zemlyanskii and G. A. Shmanenkova, "Mass-average value method for a three-dimensional boundary layer in a swirled flow," Izw. Akad. Nauk SSSR, Mekh. Zhidk Gaza, No. 1, 80 (1981).

8. D. Kh. Gan'zha, G. A. Tirskii, S. V. Utyuzhnikow, and M. O. Fridlender, "Influence of second-order boundary layer effects in hypersonic flow over slender blunt cones," Izv. Ros. Akad. Nauk, Mekh. Zhidk. Gaza, No. 4, 129 (1992).

9. S. V. Utyuzhnikov, "Numerical investigation of supersonic viscous gas flow over long blunt cones with allowance for equilibrium physicochemical effects," Izv. Ros. Akad. Nauk, Mekh. Zhidk. Gaza, No. 1, 202 (1993).

10. S. A. Vasil'evskii, G. A. Tirskii, and S. V. Utyuzhnikov, "Numerical method of solving the viscous shock layer equations," $Z h$. Vychisl. Mat. Mat. Fiz., 27, 741 (1987).

11. G. A. Tirskii, S. V. Utyuzhnikov, and N. K. Yamaleev, "Application of the small parameter method to the problem of three-dimensional viscous gas flow over bodies," Prikl. Mat. Mekh, 56, 1023 (1992).

12. L. I. Sedov, M. P. Mikhailova, and G. G. Chernyi, "Effect of viscosity and thermal conductivity on the flow behind a strongly curved shock wave," Vestn. MGU, Ser. Fiz. Mat. Nauk, No. 3, 95 (1953). 